\title{
Violencia en el Noviazgo: Diferencias de Género²
}

\author{
Jacqueline Benavides Delgado \\ Dra. En Psicología \\ Mg. Protección Infantil \\ Universidad Cooperativa de Colombia \\ Correo electrónico: jaqueline.benavidesd@campusucc.edu.co
}

Recibido: 16/05/2015

Evaluado: $16 / 05 / 2016$

Aceptado: 31/05/2016

\section{Resumen}

Objetivo: determinar las diferencias o similitudes entre la violencia que ejercen los hombres y las mujeres en las relaciones de noviazgo y sus implicaciones respecto a la equidad de género. Método: se utilizó un diseño de corte transversal, cuantitativo, no experimental; la muestra estuvo conformada por 110 jóvenes, 60 mujeres y 50 hombres cuyo promedio de edad fue de 16.9 años, estudiantes universitarios de la ciudad de Bogotá, de tres instituciones educativas, dos públicas y una privada. Como herramienta de recolección de datos se utilizó el CTS (Conflict Tactic Scale) desarrollado por Straus en 1979, con el fin de conocer los conflictos y la violencia en las relaciones intrafamiliares. Resultados: se evidenció la existencia de diferentes violencias en el noviazgo. Respecto a las diferencias entre hombres y mujeres se pudo determinar que tanto hombres como mujeres se sienten víctimas y agresores, lo cual desmitifica el noviazgo como una etapa idílica y a su vez cuestiona los roles tradicionales en los que se sitúan los hombres como agresores y las mujeres como víctimas. Conclusión: los resultados sugieren la importancia de intervenir de forma temprana con los jóvenes para modificar estas conductas.

Palabras clave

violencia, noviazgo, juventud, relaciones íntimas, género.

2 Para citar este artículo: Benavides, J. (2016). Violencia en el noviazgo: Diferencias de Género. Informes Psicológicos, 16(2), pp. 27-36. http://dx.doi.org/10.18566/infpsicv16n2a02 


\section{Dating violence: gender differences}

Abstract

Objective: to determine the differences or similarities between the violence that men and women exercise in dating relationships and their implications with respect to gender equity. Method: a cross-cutting, quantitative / non-experimental design was used; the sample was conformed by 110 youth, 60 women and 50 men whose average age was 16.9 years, college students from the city of Bogota, from three different institutions: two public and one private. The CTS (Conflict Tactic Scale), developed by Straus in 1979, was used as tool of data collection in order to learn about conflict and violence in family relations. Results: the existence of different kinds of violence in dating was revealed. Regarding the differences between men and women, it was determined that both male and female feel like victims and aggressors, which demystifies the courtship as an idyllic stage and, at the same time, questions the traditional of women as victims and men as aggressors. Conclusion: the results suggest the importance of early intervention with young people to change this behavior.

\section{Violência no namoro: diferenças de gênero}

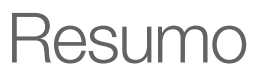

Objetivo: determinar as diferenças ou similitudes entre a violência que exercem os homens e as mulheres nas relações de namoro e suas implicações com respeito à igualdade de gênero. Método: se utilizou um desenho de corte transversal, quantitativo, não experimental; a amostra esteve conformada por 110 jovens, 60 mulheres e 50 homens cujo a média de idade foi de 16.9 anos, estudantes universitários da cidade de Bogotá, de três instituições educativas, duas públicas e uma particular. Como ferramenta de recolecção de dados se utilizou o CTS (Conflict Tactic Scale) desenvolvido por Straus em 1979, com o fim de conhecer os conflitos e a violência nas relações intrafamiliares. Resultados: se evidenciou a existência de diferentes violências no namoro. Com respeito às diferencias entre homens e mulheres se pôde determinar que tanto homens como mulheres se sentem vítimas e agressores, o qual desmitifica o namoro como uma etapa idílica e por sua vez questiona os papeis tradicionais nos que se situam os homens como agressores e as mulheres como vítimas. Conclusão: os resultados sugerem a importância de intervir de forma precoce com os jovens para modificar estas condutas.

violência, namoro, juventude, relações íntimas, gênero. 


\section{ntroducción}

El noviazgo se ha definido como una relación íntima entre dos individuos que se atraen y experimentan un tipo de amor que reúne componentes de intimidad y pasión. Esta relación tiene la finalidad de conocer la propia capacidad de dar y recibir afecto (Domínguez, García, Moreno, Zúñiga, \& Torres, 1998). Si bien, en el siglo pasado, el noviazgo era visto como una etapa idílica donde emergían los sentimientos más puros y románticos, actualmente, por los cambios sociales, tecnológicos y la diversidad de visiones sobre el género, se sabe que las relaciones de intimidad en la juventud, también son violentas (Morales \& Rodríguez, 2012; Pacheco \& Castañeda, 2013).

Algunos autores han definido la violencia en el noviazgo como la ocurrencia de actos violentos en el contexto de una relación romántica donde se combina la atracción y el abuso de poder (Corsi, Dohmen, \& Sotès, 1999; Rey, 2008, 2009, 2013). Los medios a través de los cuales se ejerce la violencia, son verbales, físicos, sexuales, económicos y también virtuales (Durán \& Martínez -Pecino, 2015). Por lo tanto, la violencia en las relaciones de intimidad en la juventud, antes del matrimonio o la convivencia, es actualmente, un problema socio-cultural (Alexander et al., 2014).

El primer estudio sobre violencia en el noviazgo realizado por Makepeace (1981), despertó el interés por el tema. Los avances posteriores mostraron la prevalencia del problema en distintos países (Edward et al., 2015) y también algunas características de este tipo de violencia en comparación con la violencia marital (Barnett, Miller- Perrin, \& Perrin, 1997; Cáceres \& Cáceres, 2006). En este sentido, se conoce que la gravedad de la violencia marital y la frecuencia de la misma superan los datos encontrados en la violencia en las relaciones de noviazgo. Echeburúa y Del Corral (1998), afirman que la violencia en las relaciones de novios equivale al 22\% de la violencia en las relaciones maritales. Otros autores señalan que la violencia en el noviazgo predice el $51 \%$ de la agresión en pareja de casados y además los porcentajes de violencia en las relaciones de novios, ascienden a $45.5 \%$ en hombres y a $46.8 \%$ en mujeres (Peña et al., 2013).

Los estudios en los EE.UU muestran un problema de grandes dimensiones entre los jóvenes, respecto a la violencia en las relaciones de noviazgo. Entre un 19-27\% de la población adolescente de los colegios han sido víctimas de violencia física o sexual, siendo la prevalencia de violencia física similar entre hombres y mujeres y la prevalencia de violencia sexual muy superior en las mujeres (Edward et al., 2015).

En España se han realizado varios estudios con jóvenes. Uno de los pioneros fue el de Gonzales y Santana (2001) con 1.146 jóvenes con edades comprendidas entre los 16 y 18 años. Este estudio se realizó utilizando la versión modificada de instrumento de Straus (1979) Conflict Tactics Scale. En este estudio se encontró que la mayoría de los jóvenes del estudio no habían experimentado violencia en sus relaciones de noviazgo. Sólo una proporción del $4.4 \%$ en las mujeres y $3.6 \%$ en los hombres manifestaron haber sido víctimas de violencia en el 
noviazgo. Otros estudios sobre el tema han sido realizados en España (Cáceres \& Cáceres, 2006; Mohamed, Herrera, \& Caicedo, 2014), donde se ha relacionado la violencia en el noviazgo y la violencia marital y la capacidad de predicción de la violencia marital a partir de la violencia en el noviazgo. América Latina no queda al margen de estas investigaciones aportando datos sobre la prevalencia del problema y aproximaciones intervenidas (Peña et al., 2013; Rey, 2008, 2009, 2013; Trezza, 2006). Estos estudios han mostrado una prevalencia de la violencia en relaciones de noviazgo que oscila entre los $35-45 \%$.

\section{Equidad de Género}

La comunidad internacional y organismos como la Organización de Naciones Unidas (ONU) han considerado la importancia de la igualdad de género y del respeto de los derechos de la mujer. Se considera que sin la igualdad entre hombres y mujeres no habrá desarrollo (Milosavljevic, 2007). Estas organizaciones han mostrado la inequidad de género en muchos campos de la vida de los hombres y las mujeres y han develado diferencias importantes en cuanto a los ingresos económicos, las oportunidades laborales, la educación, la pobreza y aspectos relacionados con la salud y el acceso a mejores condiciones de vida. Las diferencias en estos aspectos son evidentes en América Latina y en otras regiones del mundo.

Con respecto a la violencia dirigida hacia las mujeres se conoce que la mayoría de víctimas son mujeres y sus agresores hombres. Sin embargo, actualmente las investigaciones se han centrado en mostrar la perspectiva del hombre que se considera también víctima (Trujano, Martínez, \& Camacho, 2010). Específicamente en las relaciones de noviazgo se destaca la inexistencia de diferencias de género (Trujano \& Mata, 2002) a excepción de la violencia económica señalada por Rey (2013). El presente estudio busca analizar más a fondo este aspecto de las diferencias o similitudes entre la violencia que ejercen los hombres y las mujeres y sus implicaciones respecto a la equidad de género.

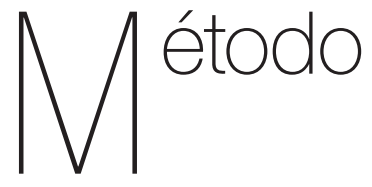

\section{Diseño}

El diseño utilizado en la investigación fue de corte transversal, cuantitativo, no experimental.

\section{Participantes}

La muestra estuvo conformada por 110 jóvenes, 60 mujeres equivalente al $54.5 \%$ de la muestra y 50 hombres (45.45\%), cuyo promedio de edad fue de 16.9 años. Todos los jóvenes eran estudiantes universitarios de la ciudad de Bogotá, de tres instituciones educativas, dos públicas y una privada. Los jóvenes que participaron en este estudio tenían una inclinación sexual heterosexual y estaban involucrados en relaciones de noviazgo de 1 año de duración como mínimo. Estos jóvenes participaron de forma 
voluntaria y firmaron un consentimiento informado.

\section{Instrumentos}

Como herramienta de recolección de datos se utilizó el CTS (Conflict Tactic Scale) desarrollado por Straus en 1979, con el fin de conocer los conflictos intrafamiliares y la violencia en las relaciones familiares. Este instrumento consiste en 15 items diseñados para medir tres formas de resolver los conflictos en pareja: racionalidad, violencia física y violencia verbal. Las repuestas a cada pregunta se registran en escala de Likert y el participante responde acerca de su conducta y de la conducta de su pareja. El instrumento utiliza una escala de Likert con 6 opciones de respuesta (1 vez, 2-5 veces, 6-10, 1120, más de 20 y nunca). La versión que se utilizó en la aplicación correspondió al instrumento en español validado por Pérez (2000), al cual se le hicieron los ajustes derivados de un pilotaje con 30 jóvenes de la ciudad de Bogotá.

El instrumento CTS permite identificar conductas físicamente agresivas y catalogarlas como leves, moderadas o graves. Otro de los aportes del instrumento es la capacidad que tiene de evaluar la percepción que se tiene de la pareja respecto a la utilización de las conductas agresivas y también la auto-evaluación que cada persona hace de éstas. De igual forma, a través de la aplicación de CTS se pueden clasificar 4 tipos de conductas: racionales, agresión verbal, agresión física y agresión sexual, y además identificar en qué medida la persona que responde ha sido víctima o agresora.

\section{Procedimiento}

En cada una de las tres instituciones educativas de la ciudad de Bogotá, se realizó una charla informativa donde se explicó a los jóvenes los alcances de la investigación. Se hizo especial énfasis en la confidencialidad y anonimato de los resultados. De igual manera, se entregó el consentimiento informado explicándoles la voluntariedad de su decisión, para que ellos pudieran libremente participar en el estudio. Las aplicaciones del CTS se realizaron de manera grupal en cada una de las instituciones educativas.

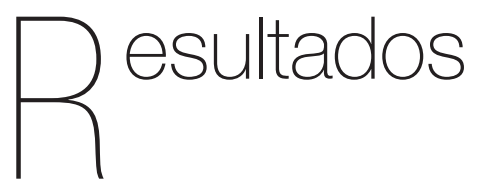

Los resultados que se presentan a continuación se organizan de acuerdo a las preguntas de investigación formuladas. La primera de las preguntas buscaba identificar las diferencias entre hombres y mujeres respecto a la utilización de la violencia física, sexual o verbal con sus parejas. Se realizó una prueba t para muestras independientes.

Con un alfa de .05 se encontraron diferencias significativas en las variables: Victimización a través de la violencia física leve $(.01 ; p<.05)$. De igual forma, se encuentran diferencias significativas entre hombres y mujeres respecto a la agresión sexual por parte de hombres más que de mujeres (.04; $p<.05)$. En la Tabla 1 se presenta los promedios obtenidos en la prueba y las diferencias significativas. 
Tabla 1.

Violencia Física

\begin{tabular}{cccc}
\hline Variable & X mujer & X hombre & $\mathrm{P}<.05$ \\
\hline Víctima & & & \\
\hline Leve & 1.0 & 1.7 & $.01^{*}$ \\
Moderada & .2 & .3 & .13 \\
Grave & .01 & .00 & .06 \\
\hline Agresor & & & \\
\hline Leve & 1.3 & 1.7 & .49 \\
Moderada & .3 & .2 & .13 \\
Grave & .03 & .06 & .33 \\
\hline Agresión Verbal & & & \\
\hline Víctima & 3.9 & 4.7 & .48 \\
Agresor & 4.2 & 4.7 & .22 \\
\hline Agresión Sexual & & & \\
\hline Víctima & .10 & .14 & .29 \\
Agresor & .08 & .16 & .04 \\
\hline
\end{tabular}

Respecto a los otros tipos de violencia no se reportaron diferencias significativas. Los porcentajes de frecuencias demuestran que el nivel de violencia en las parejas de novios adolescentes varía según el tipo de violencia. El 92.7\% de los jóvenes ha estado en relaciones de pareja en las cuales se han sentido víctimas de agresión verbal. Un 10\% de los jóvenes han tenido conductas sexualmente agresivas con sus parejas y un $2.72 \%$ han sido perpetradores de conductas calificadas como agresión física severa, las cuales incluyen utilización de armas corto punzantes, amenaza con armas de fuego entre otros. En estos casos de violencia extrema, el porcentaje de hombres es del $4 \%$ mientras que en las mujeres es de $1.6 \%$. Respecto a la agresión sexual se puede determinar que el $14 \%$ de los hombres han reportado agresión sexual con su pareja, como se observa en la Tabla 2.
Los resultados que se muestran en la Tabla 2, plantean algunas diferencias porcentuales respecto a la posición de víctima de los hombres y las mujeres. En este sentido se observa que los hombres se han sentido víctimas de violencia sexual en un 10\%; de igual manera, reportan haber sido víctimas de violencia física, donde la violencia leve es la más frecuentemente reportada, junto con la agresión verbal. En esta Tabla se muestra cómo las mujeres se reportan como agresoras, tanto de violencia física, como sexual y verbal.

Tabla 2

Porcentaje de Respuestas por Género

\begin{tabular}{cccc}
\hline Variables & $\begin{array}{c}\text { Frecuencia } \\
\text { mujeres }\end{array}$ & $\begin{array}{c}\text { Frecuencia } \\
\text { hombres }\end{array}$ & $\begin{array}{c}\text { Frecuencia } \\
\text { total }\end{array}$ \\
\hline Víctimas & & & \\
\hline Verbal & $91.6 \%$ & $94 \%$ & $92.7 \%$ \\
Física Leve & $53.3 \%$ & $56 \%$ & $54.5 \%$ \\
Física Moderada & $13.3 \%$ & $20 \%$ & $16.3 \%$ \\
Física Severa & $1.6 \%$ & $0 \%$ & $.90 \%$ \\
\hline Agresor & & & \\
\hline Agresión Verbal & $90 \%$ & $90 \%$ & $90 \%$ \\
Física Leve & $51.6 \%$ & $42 \%$ & $47.2 \%$ \\
Física Moderada & $13.3 \%$ & $16 \%$ & $14.5 \%$ \\
Física Severa & $1.6 \%$ & $4 \%$ & $2.72 \%$ \\
\hline Abuso Sexual & & & \\
\hline Víctima & $8.3 \%$ & $10 \%$ & $10 \%$ \\
Agresor & $6.6 \%$ & $14 \%$ & $10 \%$ \\
\hline
\end{tabular}

Al utilizar la prueba de correlación de Pearson $\mathrm{p}<$.05, se encontró una correlación positiva entre la duración de la relación y la victimización a través de violencia física leve y del rol de agresor a través de violencia física leve y moderada. En este sentido, a mayor tiempo de permanencia en la pareja, mayores los niveles de violencia física moderada y leve. Además con el análisis de correlación de Pearson, 
se encontró un índice de correlación positiva para las variables estrato socioeconómico y violencia física leve víctima y física moderada agresor (Ver Tabla 3).

Tabla 3.

Correlación Entre la Duración y Tipos de Violencia

\begin{tabular}{lcccc}
\hline Agresor & $\begin{array}{c}\text { Victima } \\
\text { Violencia } \\
\text { Física } \\
\text { Leve }\end{array}$ & $\begin{array}{c}\text { Agresor } \\
\text { Violencia } \\
\text { Física } \\
\text { Leve }\end{array}$ & $\begin{array}{c}\text { Violencia } \\
\text { Física } \\
\text { Moderada } \\
\text { Agresor }\end{array}$ \\
\hline Duración & $.209^{*}$ & $.190^{*}$ & $.274^{*}$ & $.224^{*}$ \\
\hline${ }^{*} p<.05$ & & & & \\
& & & &
\end{tabular}

Utilizando la prueba de correlación de Pearson $\mathrm{p}<.05$, se encontró una correlación positiva entre la duración de la relación y la victimización a través de violencia física leve y del rol de agresor a través de violencia física leve y moderada. En este sentido, a mayor tiempo de permanencia en la pareja, mayores los niveles de violencia física moderada y leve (Ver Tabla 4).

Tabla 4.

Correlación Entre la Duración y Tipos de Violencia

\begin{tabular}{ccccc}
\hline Agresor & $\begin{array}{c}\text { Victima } \\
\text { Violencia } \\
\text { Física } \\
\text { Leve }\end{array}$ & $\begin{array}{c}\text { Agresor } \\
\text { Violencia } \\
\text { Física } \\
\text { Leve }\end{array}$ & $\begin{array}{c}\text { Violencia } \\
\text { Física } \\
\text { Moderada } \\
\text { Agresor }\end{array}$ \\
\hline Duración & $.209^{*}$ & $.190^{*}$ & $.274^{*}$ & $.224^{*}$ \\
\hline
\end{tabular}

${ }^{*} p<.05$

Con el análisis de correlación de Pearson, se encontró un índice de correlación negativa, a mayor estrato menor violencia física leve víctima y física moderada agresor. (Ver Tabla 5).

Tabla 5.

Correlación entre estrato socio-económico y tipos de violencia

\begin{tabular}{ccc}
\hline & Física-leve-vict & Física-mod-agres \\
\hline Estrato & $-.237^{\star}$ & $-.234^{\star}$ \\
\hline
\end{tabular}

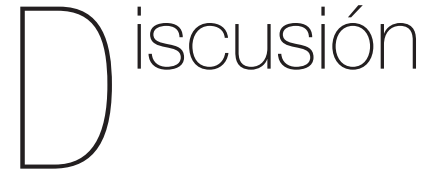

La imagen del noviazgo como una etapa de escasos conflictos puede ser revaluada a través de los datos obtenidos en esta investigación. Por una parte, los jóvenes reconocen haber estado involucrado en relaciones de noviazgo donde la forma de resolver los conflictos atraviesa por prácticas violentas verbales, físicas y sexuales. De acuerdo a los objetivos del estudio, los resultados muestran que no hay diferencia significativa en el uso de la violencia verbal, física severa y física moderada. Estos datos corroboran los encontrados en la mayoría de los estudios en este campo (González \& Santana, 2001; Pérez, 2000; Rey, 2008, 2009, 2013). Sin embargo, la utilización de la violencia física leve parece ser una práctica que se está generalizando por parte de las mujeres jóvenes.

De otro lado, los resultados obtenidos en esta investigación superan las estadísticas reportadas por Rey (2013) quien encontró un porcentaje de producción de violencia y de experiencia de violencia del 85\% en una muestra de 902 adolescentes y jóvenes colombianos. En el presente estudio el $94 \%$ de la muestra reportó haber experimentado violencia en el noviazgo, siendo agresor o víctima. Estas cifras demuestran la existencia de un fenómeno muy generalizado en la población joven de la ciudad de Bogotá.

De otro lado, este estudio hace evidente que la imagen de hombre incapaz de denunciar las agresiones de las mujeres por temor a ser calificado de débil, está 
cambiando. De igual manera, las mujeres parecen estar abandonando su imagen de víctimas y asumiendo una actitud más crítica sobre su propio comportamiento violento. Éste es sin lugar a dudas un paso fundamental que permite ver una dinámica de relaciones menos atada a los roles sexuales tradicionales. Los resultados obtenidos en esta investigación apoyan este argumento demostrando que un porcentaje elevado de hombres (94\%), se han sentido al menos 1 vez en el último año víctimas de maltrato verbal por parte de su pareja femenina.

En el análisis de estos resultados se destaca un porcentaje del 90\% de mujeres que reconocen haber agredido verbalmente al menos una vez en el último año a su pareja masculina. La agresión leve como empujarse, pegarse suavemente, también reportan datos similares en hombres y mujeres. A diferencia de las relaciones maritales, en las relaciones de noviazgo las agresiones graves no son muy frecuentes (2.72\%).

Por otra parte, es interesante analizar la correlación positiva existente entre la duración de la relación y la presencia de violencia física leve. Podría plantearse que a medida que pasa el tiempo se permiten más acercamientos físicos entre las parejas, y se diluye cierto respeto que lleva a una mayor agresión física y verbal. Este fenómeno se puede asociar de manera muy clara con el aumento de la violencia física y verbal en las parejas que conviven y tienen relaciones estables (Corsi, 2004).

Los resultados apuntan a varias conclusiones. Se muestra cómo la violencia en las relaciones inician en el noviazgo y se conoce que a mayor tiempo de relación mayor violencia. Este estudio muestra el fenómeno de equidad entre hombres y mujeres que puede analizarse desde diferentes miradas. Por una parte, existe una conciencia de victimización en los hombres jóvenes, lo cual permite una apertura desconocida anteriormente, donde los hombres no hablaban de sus emociones ni de la violencia que se ejercía hacia ellos por parte de las mujeres. Este estudio identifica el tiempo de relación como una variable que potencia la violencia. En este sentido, la intervención temprana de la violencia marital es inminente, mediante programas de prevención de la violencia marital, de la violencia en el noviazgo y de una convivencia en paz, se hacen necesarios.

Estos resultados también dejan abierta la posibilidad de nuevas investigaciones que combinen elementos cualitativos, donde se profundice en los motivos, los sentimientos, los pensamientos y conductas que se derivan de la violencia que aquí se expone.

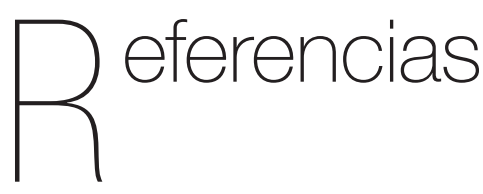

Alexander, C., Hutchison, N., Clougher, M., Davis, H., Shepler, D., \& Ambroise, Y. (2014). Adolescent dating violence: Application of a U.S primary prevention program in St. Lucia. Journal of Counseling \& Development, 92, 488-498.

Barnett, O., Miller-Perrin, C., \& Perrin. (1997). Family violence across the lifespan. An Introduction. Londres: Sage. 
Cáceres, A., \& Cáceres, J. (2006). Violencia en relaciones intimas en dos etapas evolutivas, International Journal of Clinical and Health Psychology, 6(2), 271-284.

Corsi, J. (2004). Maltrato y abuso en el ámbito doméstico. Buenos Aires: Paidós.

Corsi, J., Dohner, M., \& Soles, M.A. (1999). Violencia masculina en la pareja: Una aproximación al diagnóstico y los modelos de intervención. Argentina: Paidós.

Domínguez, A., García, D., Gutiérrez, V., Moreno, A., \& Zúñiga, K. (1998). Violencia en el noviazgo. Revista Conducta, 7(14), 37-39.

Durán, M., \& Martínez-Pecino, R. (2015). Ciberacoso mediante teléfono móvil e internet en las relaciones de noviazgo entre jóvenes. Comunicar, 44(XXII), 159-167.

Echeburua, E., Del Corral, P., Sarasua, B., \& Zubizarreta, I. (1998). En E. Echeburua, \& P. Del Corral (1998). Manual de Violencia Intrafamiliar. Barcelona: Siglo XXI.

Edward, K., Sylaska, K., Barry, J., Muynihan, M., Banyard, U., Cohn, E., Walsh, W., \& Ward, S. (2015). Physical dating violence, sexual violence, and unwanted pursuit victimization: A comparison of incidence rates among sexual minority and heterosexual colleges students. Journal of Interpersonal Violence, 30(4), 560-600.

González, R., \& Santana, J. D. (2001).Violencia en parejas jóvenes: Analisis y prevención. Madrid: Piramide.

Makepeace, J.M. (1981). Courtship violence among college student. Family Relations, 30, 97-102.
Milosavljevic, V. (2007). Estadísticas para la equidad de género. Magnitudes y tendencias en América Latina. Santigo de Chile: ONU.

Mohamed, L., Herrera, L., \& Caicedo, S. (2014). Violencia de pareja en jóvenes estudiantes universitarios de diferente origen cultural. Revista de Educacao e Humanidades, 5, 223-236.

Morales, N., \& Rodríguez, V. (2012). Violencia en relaciones de noviazgo. Revista Puertorriqueña de Psicología, 23, 57-90.

Pacheco, K., \& Castañeda, J. (2013). Hombres receptores de violencia en el noviazgo. Avances en Psicología, 21(2), 207-221.

Peña, F., Zamorano, B., Hernández, G., Hernández, M., González, J., Vargas, J.I., \& Parra, V. (2013). Revista Costarricense de Psicología, 32(1), 27-40.

Pérez, M. (2001). Prevalencia de agresiones verbales y físicas entre parejas: La importancia de los factores demográficos, la identidad de género y la experiencia de ira en dos muestras de hombres y mujeres residentes en Bogotá. Tesis de Grado. Universidad de los Andes.

Rey, C.A. (2008). Prevalencia, factores de riesgo t problemática asociada con la violencia en el noviazgo: Una revisión de la literatura. Avances de Psicología Latinoamericana, 26(2), 227-241.

Rey, C.A. (2009). Maltrato de tipo físico, psicológico, emocional, sexualy económico en el noviazgo: Un estudio exploratorio. Acta Colombiana de Psicología, 12(2), 27-36. 
Rey, C. (2013). Prevalencia y tipos de maltrato en el noviazgo en adolescentes y adultos jóvenes. Terapia Psicológica, 3(2), 143-154.

Trezza, F. (2006). Alerta por violencia en parejas jóvenes. Revista Venezolana de Estudios de la Mujer, 3(1), 141-146.

Trujano, P., Martínez, A., \& Camacho, S. (2010). Varones víctimas de violencia doméstica: Un estudio exploratorio acerca de su percepción y aceptación. Diversitas: Perspectivas Psicológicas, 6(2), 339-254.
Trujano, P., \& Mata, E. (2002). Relaciones violentas en el noviazgo: Un estudio exploratorio. Revista Psicológica Conductual, 10(2), 389-408.

Trujano, P., \& Mendoza, S. (2003). Violencia doméstica: estudio exploratorio acerca de su percepción y aceptación. Revista Iztapdapa, 54, 231-244. 